\title{
Contribution of Individual Risk Factor Changes to Reductions in Population Absolute Cardiovascular Risk
}

\author{
Thomas Cochrane, ${ }^{1}$ Rachel Davey, ${ }^{1}$ Christopher Gidlow, ${ }^{2}$ Zafar Iqbal, ${ }^{3}$ Jagdish Kumar, \\ Yvonne Mawby, ${ }^{3}$ and Ruth Chambers ${ }^{3}$ \\ ${ }^{1}$ Centre for Research and Action in Public Health, Faculty of Health, University of Canberra, ACT 2601, Australia \\ ${ }^{2}$ Centre for Sport Health and Exercise Research, Staffordshire University, Leek Road, Stoke-on-Trent ST4 2DF, UK \\ ${ }^{3}$ Stoke-on-Trent City Council Public Health Directorate, Civic Centre, Glebe Street, Stoke-on-Trent ST4 1HH, UK \\ Correspondence should be addressed to Christopher Gidlow; c.gidlow@staffs.ac.uk
}

Received 12 February 2014; Accepted 13 May 2014; Published 5 June 2014

Academic Editor: Mangesh S. Pednekar

Copyright (C) 2014 Thomas Cochrane et al. This is an open access article distributed under the Creative Commons Attribution License, which permits unrestricted use, distribution, and reproduction in any medium, provided the original work is properly cited.

\begin{abstract}
Background. Few studies have investigated individual risk factor contributions to absolute cardiovascular disease (CVD) risk. Even fewer have examined changes in individual risk factors as components of overall modifiable risk change following a CVD prevention intervention. Design. Longitudinal study of population CVD risk factor changes following a health screening and enhanced support programme. Methods. The contribution of individual risk factors to the estimated absolute CVD risk in a population of high risk patients identified from general practice records was evaluated. Further, the proportion of the modifiable risk attributable to each factor that was removed following one year of enhanced support was estimated. Results. Mean age of patients (533 males, 68 females) was 63.7 (6.4) years. High cholesterol (57\%) was most prevalent, followed by smoking (53\%) and high blood pressure (26\%). Smoking (57\%) made the greatest contribution to the modifiable population CVD risk, followed by raised blood pressure (26\%) and raised cholesterol (17\%). After one year of enhanced support, the modifiable population risk attributed to smoking (56\%), high blood pressure (68\%), and high cholesterol (53\%) was removed. Conclusion. Approximately 59\% of the modifiable risk attributable to the combination of high blood pressure, high cholesterol, and current smoking was removed after intervention.
\end{abstract}

\section{Introduction}

Progress in understanding the multifactorial nature of cardiovascular disease and the cumulative effect of combinations of risk factors has evolved rapidly in recent years since the pioneering research of Anderson and colleagues [1] as part of the Framingham Heart study [2]. This work is given added impetus because of the growing global burden of chronic diseases as the world's population both increases and ages [3-5]. The latter emphasises the need for greater efforts to be placed on modifying lifestyles to prevent, or at least forestall, the occurrence of some risk factors in the first place. With this objective in mind, the Department of Health in England introduced the NHS Health Checks programme in 2009 [6]. We have demonstrated previously that a local implementation of this national policy in Stoke on Trent led to around a $10 \%$ decrease in mean population cardiovascular disease
(CVD) risk (32.55\% to $29.57 \%$ ) in an established high risk population [7]. In this paper, we investigate the contribution of change in individual risk factors to the overall change in absolute cardiovascular risk.

\section{Methods}

2.1. Recruitment of Practices and Patients. Full details of our study design have been given in our previous publications $[7,8]$. Here we report on further detailed analysis of the individual risk factor contributions to the changes in absolute CVD risk in the study sample of 601 patients recruited from 35 general practices in Stoke on Trent, a mid-sized industrial city in the West Midlands region of England. Electronic medical records of 118,710 (57,468 female, 61,242 male) patients were screened for CVD risk, of whom 10,483 
(8,521 male, 1962 female) were estimated to have a CVD risk $\geq 20 \%$ over the next 10 years. Diabetic patients were excluded from the study at recruitment as there was a separate diabetesspecific management programme underway in primary care across the city. Our original trial included two groups, 365 recruited to a NHS Health Check only group and 236 to a NHS Health Check plus additional lifestyle support group [7]. Since there was no significant difference in mean population CVD risk between the two groups either at baseline or at the one year follow-up point, we have merged the data and consider the contribution of individual risk factor changes to the overall population change in CVD risk.

\subsection{Absolute Cardiovascular Risk Estimation. Absolute CVD} risk was based on the Framingham 10-year CVD risk as recommended in the Joint British Societies' Guidelines [9]. In the absence of established diabetes or confirmed left ventricular hypertrophy, the estimated CVD risk for an individual patient is based on two nonmodifiable risk factors, age and gender, and three modifiable risk factors, systolic blood pressure, total to high density lipoprotein (HDL) cholesterol ratio, and smoking status. The absolute CVD risk for an individual patient is estimated as the percentage chance that that patient will experience a CVD event over the next 10 years. Using the original parametric model and associated regression coefficients, it is possible to partition out the individual risk factor contributions to the overall absolute CVD risk estimated for each patient and to examine the mechanisms for population risk reduction in more detail.

In the risk estimator used in this study, the component of risk due to the nonmodifiable combination of gender and age, $C_{\mathrm{GA}}$, is governed by an equation of the form

$$
\begin{aligned}
C_{\mathrm{GA}}= & 1.2146 * \text { gender }+1.8433 * \ln (\text { age }) \\
& -0.3668 * \text { gender } * \ln (\text { age }),
\end{aligned}
$$

where gender is coded 1 for males and 0 for females and $\ln$ represents the natural logarithm function.

The modifiable components, $C_{\mathrm{SBP}}, C_{\mathrm{C}}$, and $C_{\mathrm{S}}$, due, respectively, to systolic blood pressure, total to HDL cholesterol ratio, and smoking are governed by equations of the form

$$
\begin{aligned}
& C_{\mathrm{SBP}}=1.4032 * \ln (\text { systolic blood pressure }), \\
C_{\mathrm{C}}= & 0.5390 \\
& * \ln (\text { total cholesterol to HDL cholesterol ratio }), \\
& C_{\mathrm{S}}=0.3899 * \text { Smoker }
\end{aligned}
$$

where Smoker is coded 1 for a current smoker, 0 otherwise.

We have used the above formulae to calculate the component of absolute CVD risk attributable to the combined nonmodifiable risk factors and each of the three modifiable risk factors individually. For the risk factors systolic blood pressure and total to HDL cholesterol ratio, where a threshold needs to be exceeded before the factor is considered a risk, we have further divided the risk into an unmodifiable and a modifiable component. For example, a patient is only
TABLE 1: Baseline characteristics of sample population.

\begin{tabular}{lcc}
\hline Characteristic & Category & $N(\%)$ \\
\hline \multirow{2}{*}{ Gender } & Female & $68(11.3 \%)$ \\
& Male & $533(88.7 \%)$ \\
Ethnic group & White & $581(96.7 \%)$ \\
& Other & $20(3.3 \%)$ \\
Socioeconomic status & Deprived & $288(48 \%)$ \\
& Intermediate & $195(32 \%)$ \\
& More affluent & $118(20 \%)$ \\
\hline Measure & & Mean (SD) \\
Age (years) & & $63.7(6.4)$ \\
Systolic blood pressure $(\mathrm{mmHg})$ & & $145(16.7)$ \\
Total/HDL cholesterol ratio & & $4.8(1.1)$ \\
Weight (kg) & & $83.6(14.1)$ \\
Body mass index $\left(\mathrm{kgm}^{-2}\right)$ & & $28(4.5)$ \\
\hline
\end{tabular}

${ }^{\mathrm{a}}$ Based on the English Indices of deprivation 2010.

considered to have a modifiable blood pressure component of CVD risk if systolic blood pressure exceeds $140 \mathrm{mmHg}$. Thus systolic blood pressure up to $140 \mathrm{mmHg}$ was taken as the unmodifiable part and anything above this threshold was taken as the modifiable component. Similarly, a patient is only considered to have a modifiable cholesterol component of CVD risk if total to HDL cholesterol ratio exceeds 4.5.

2.3. Changes in Modifiable Risk. For each modifiable risk factor, the change in risk was calculated as a proportion of the modifiable risk attributable to that factor so that an estimate of the proportion of modifiable risk reduced or added to could be derived for each patient. The mean population proportions were then compared with zero (i.e., no change in risk factor), using the one-sample $t$-test, to determine whether there was a significant net benefit of the intervention and, if so, to derive $95 \%$ confidence intervals for the proportion of modifiable risk reduced by the intervention. The significance level was set at $P<0.05$.

\section{Results}

3.1. Characteristics of Sample Population. The baseline characteristics of the sample population are summarised in Table 1.

As can be seen from the table, this population was predominantly of older age, male, White, and of low or intermediate socioeconomic status. The risk factor profile of this high CVD risk population is shown in Table 2.

The most prevalent of the established CVD risk factors was high cholesterol (57\%) followed by smoking (53\%) and high blood pressure (26\%). It is also worth noting that overweight or obesity was highly prevalent in this sample (76\%), though this risk factor is not included explicitly in the currently (at the time of conducting this research) recommended CVD population risk estimator.

3.2. Components of Absolute CVD Risk. Figure 1 summarises the various components in the absolute CVD risk estimation. 
TABLE 2: Risk factor profile of sample population.

\begin{tabular}{lcc}
\hline Risk factor & Definition & $N(\%)$ \\
\hline High blood pressure & SBP $\geq 140$ and DBP $\geq 90$ & $158(26 \%)$ \\
High cholesterol & TC/HDL ratio $\geq 4.5$ & $344(57 \%)$ \\
Current smoker & Yes & $319(53 \%)$ \\
Overweight & BMI $\geq 25$ and BMI $<30$ & $291(48 \%)$ \\
Obese & BMI $\geq 30$ & $166(28 \%)$ \\
\hline
\end{tabular}

SBP: systolic blood pressure $(\mathrm{mmHg})$; DBP: diastolic blood pressure; TC/HDL: total to high density lipoprotein cholesterol ratio; BMI: body mass index $\left(\mathrm{kgm}^{-2}\right)$.

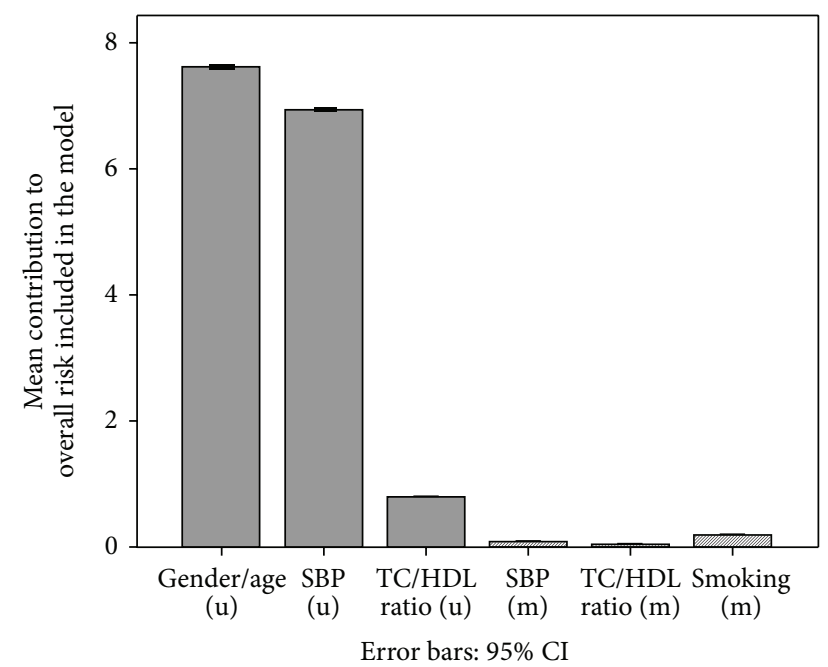

FIgUre 1: Components of absolute CVD risk. SBP: systolic blood pressure; TC/HDL: total to HDL cholesterol ratio; (u): unmodifiable; (m): modifiable.

The unmodifiable $(\mathrm{u})$ components are shown on the left and the modifiable $(\mathrm{m})$ components are shown on the right. It can be seen that the calculation is dominated by the unmodifiable risk components. Of the modifiable risk, smoking, at 57\%, made the greatest contribution to total modifiable population risk followed by blood pressure, at $26 \%$, and cholesterol, at $17 \%$.

3.3. Changes in Absolute CVD Risk due to NHS Health Checks Intervention. The proportion of the modifiable risk at baseline that was reduced for each of the primary risk factors is summarised in Table 3. Overall, approximately $59 \%$ of the modifiable risk at baseline was reduced after one year of intervention. Figure 2 illustrates the cumulative benefit of treating multiple risk factors simultaneously.

The first bar shows the negative effect of age increasing by 1 year (age and gender component of risk) (RF1). This was more than compensated for by the beneficial change in the systolic blood pressure component (RF2), with additional benefit when reduction in TC/HDL ratio (RF3) and smoking components (RF4) were added.

There was wide variation in individual patient reduction in \% CVD risk, ranging from -16.4 to +26.2 but no
TABLE 3: Proportion of modifiable risk reduced for each risk factor.

\begin{tabular}{lccc}
\hline & $\begin{array}{c}\text { Proportion of } \\
\text { modifiable risk } \\
\text { reduced (\%) }\end{array}$ & $95 \%$ CI & $N$ \\
\hline Smoking & 56 & $51-62$ & $319^{* * *}$ \\
Systolic blood pressure & 68 & $58-77$ & $322^{* * *}$ \\
Total to HDL cholesterol ratio & 53 & $42-64$ & $309^{* * *}$ \\
\hline
\end{tabular}

$N$ : number of patients with this modifiable risk; ${ }^{* * *} P<0.001$.

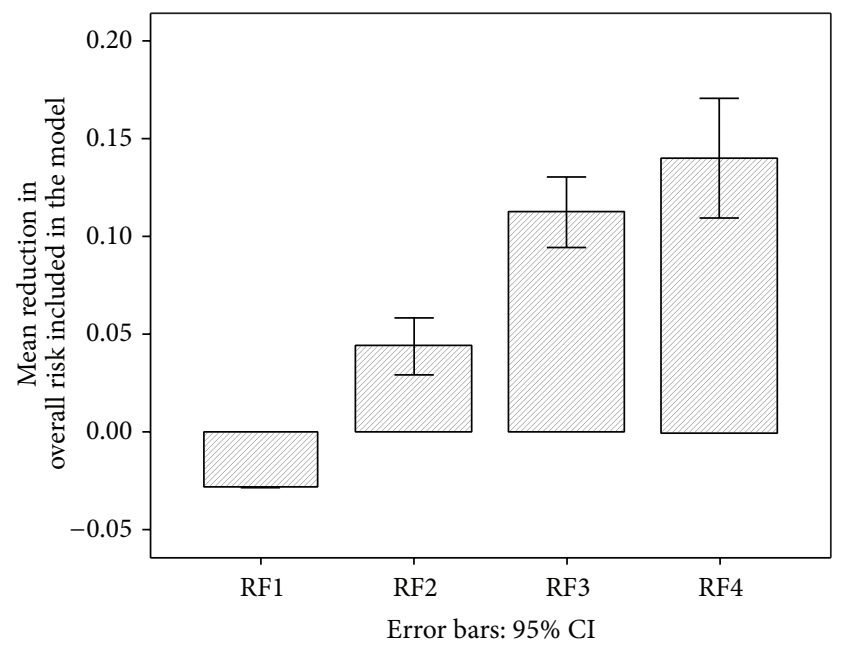

FIGURE 2: Cumulative effects of multiple risk factors. RF1: gender/age; RF2: + SBP; RF3: + TC/HDL; RF4: + smoking.

real pattern characterising those who showed no change or increased their risk and those who reduced their risk. We performed binary logistic regression analysis on the dichotomous outcome variable $(0=$ no change or worse, $1=$ reduction in CVD risk) on predictors of age at baseline (expressed as decade $=$ age/10 in order to obtain an odds ratio for a meaningful change in age) and categories of gender and smoking status, high blood pressure, high cholesterol, and obesity at baseline. Just two factors made a significant explanatory contribution in the model. Older patients and those who had a high blood pressure at baseline were more likely to show a reduction in CVD risk. However, the overall predictive power of the model was low, Nagelkerke's $R^{2}=$ .063 .

3.4. Estimates of Major Vascular Disease Events Avoided in Stoke on Trent. The mean population 10-year CVD risk for females decreased from $26 \%$ at baseline to $21.2 \%$ after one year of intervention and for males mean population CVD risk decreased from $33.4 \%$ to $30.3 \%$. Furthermore, the proportion of the male population estimated to be at high risk of experiencing a CVD related event was 13.9\% (95\% CI, 13.6-14.2), while that for females was 3.4\% (95\% CI, 3.3-3.6). Extrapolating these data to the whole of the at risk population of Stoke on Trent ( 150,000, 77,500 males, 72,500 females), estimated $\sim 450$ serious CVD events could be prevented over 10 years. 


\section{Discussion}

4.1. Key Findings. The multifactorial risk factor approach to population CVD risk reduction offers significant advantage over the single risk factor approach and has the potential to reduce the incidence of major vascular disease related events, though this would need to be confirmed in prospective longer term studies. Moreover, the success of routine screening of electronic medical records allows for a more proactive and more precisely targeted approach to the management of population CVD risk than has been available until now.

Fifty-seven percent of the modifiable CVD risk in our sample population was attributable to smoking, $26 \%$ to high blood pressure, and $17 \%$ to a high TC/HDL cholesterol ratio. The INTERHEART study [5] of potentially modifiable risk factors associated with myocardial infarction reported population attributable risks of $35.7 \%, 49.2 \%$, and $17.9 \%$ for smoking, apolipoprotein B to apolipoprotein A1 ratio, and history of hypertension, respectively. Whilst the relative order of the three risk factors differs, reflecting differences in population demographics, specific measures used, and lifestyles in Stoke on Trent compared to the global population sampled in the INTERHEART study, the dominant importance of these three risk factors in the progress of cardiovascular disease is confirmed.

More importantly, perhaps, in the context of this research, approximately 59\% of the modifiable CVD risk in this high risk population was removed after just one year of intervention. If replicated nationally this would represent a significant public health gain and should make a valuable contribution to reducing the burden of chronic diseases, of which the vascular disease cluster remains dominant $[3,4,10]$. This $59 \%$ reduction in modifiable CVD risk represents a substantial proportion of the $80 \%$ reported to be avoidable collectively using the nine lifestyle-related risk factors considered in the INTERHEART study. Furthermore, the relative risk, comparing estimated baseline mean population CVD risk with that after one year of follow-up, was 0.91 (29.57 versus 32.55), which is close to the value of 0.9 used by Barton et al. [11] in their modelling study to develop National Institute of Health and Clinical Excellence guidance on a public health programme aimed at preventing CVD in whole populations. Thus, our findings provide support for the value of the NHS Health Checks component of national policy for preventing CVD in the population of Stoke on Trent. This, of course, is only one part of the whole story and more needs to be done to reduce the average levels of established risk factors across the whole population, not just those already considered to be at high CVD risk [12-16]. Nonetheless, the observed changes in a deprived ex-industrial city with a poor health profile [17] such as Stoke on Trent are encouraging.

Another key finding from this research relates to the importance of unmodifiable factors (in particular gender and age) included in the risk estimator. For example, risk is unavoidably inflated for males and people of older age. This may explain why our sample population (identified on the basis of having a CVD risk of $\geq 20 \%$ ) was predominantly male ( $~ 8: 1$ ratio of males to females) and of older mean age than would be expected from the profile of CVD events in the whole population. This may be a more important consideration than has been recognised hitherto and represents a serious limitation of the Framingham risk estimator approach to population CVD risk reduction. It is possible that our study participants included older people who, although they had a confirmed CVD risk $\geq 20 \%$ as per national guidelines, actually had a modifiable level of risk that was lower than that of younger people with an absolute risk $<20 \%$. This has implications for the overall efficiency and/or effectiveness of national policy and the optimum reduction in burden of disease achievable across the whole population. From a prevention perspective, it would be better if the risk estimator included only modifiable factors. In addition, where absolute measures are used in the calculation, it would be better if these were expressed as deviations from an established norm or only considered for values above (or below, depending on the direction of the relationship of the measure with risk) a set threshold, for example, $140 \mathrm{mmHg}$ for systolic blood pressure.

These latter issues raise questions about the continuing suitability of the Framingham-based approach as the primary tool for use in the prevention of cardiovascular disease. The Framingham equations were developed from the research carried out in the town of Framingham, Massachusetts, in the United States, beginning in 1948 and still continuing today. Whilst there is no denying the importance of the factors included in the original equations, they form a limited subset of the factors now known to predispose individuals to cardiovascular disease risk and do not include factors such as obesity [18], socioeconomic deprivation [19, 20], and psychosocial stress $[21,22]$. In addition, lifestyles and global socioeconomic circumstances have changed markedly since the formative period over which the original equations were developed. Furthermore, electronic communication and access to linked demographic and health care information have improved dramatically in recent years, making it feasible to develop predictive models including more locally representative populations and additional relevant variables as well as more sophisticated statistical methods, not available to the original Framingham researchers. The latter might also include multilevel approaches which allow effects of higher level factors, such as the effects of neighbourhood environments, to be included in predictive models [23-25]. Several alternative approaches have been proposed and these have been reviewed recently [26]. However, there is no strong evidence, as yet, of a significant advantage of these newer methods over the original Framingham equations [27]. Perhaps it is time for a more concerted global collaborative effort to develop a 21st century upgrade to the original Framingham equations. That said, a more important issue, as noted by Cooney et al. [26], is the underutilisation of multifactorial risk estimators and prevention guidelines in clinical practice and across the wider community.

Although not without some challenges, the roll-out of software (Oberoi Clinical Observations, Oberoi Consulting, Derby, UK) for the management of CVD risk across the majority of general practices in Stoke on Trent was achieved relatively smoothly and at reasonable cost (approximately $\sim £ 500$ per practice per annum). This raises the possibility of a performance improvement in health care that could actually 
pay for itself (and more) since the societal saving in avoiding just one major cardiovascular disease event is significantly more than this. If one adds the potential to manage other chronic conditions using a similar approach, then the gains for public health could be even greater. Blumenthal [28] has advocated the setting up of health improvement communities across the United States, whereby, in return for financial, technical, and regulatory support, stakeholders in participating communities should be involved in accountablecare arrangements. In essence, the programme that we have implemented in Stoke on Trent was an embryonic trial of this basic idea. The enabling tools for the approach were improved primary care through greater efforts to screen patients, to inform them, to motivate them to take their medications (including for multiple risk factors), and to support them within their local community to make lifestyle changes that should benefit their health; funding was made available using payment for service through a local enhanced service arrangement and support for free access to additional services and a software support system that allowed each practice's electronic medical records to be searched and a cardiovascular disease risk profile to be generated, from which appropriate patients could be selected for further follow-up.

4.2. What Is Already Known on This Topic. Multifactorial risk factor interventions for the prevention of cardiovascular disease have been shown to be effective in well-controlled, well-funded randomised controlled trials.

Greater emphasis on prevention has been advocated to address the growing burden of chronic disorders as the world's population both grows in number and ages.

4.3. What This Study Adds. Routine screening of electronic medical records in general practice to estimate population CVD risk is feasible and affordable.

Coordinated high risk screening and additional support to reduce multiple risk factors simultaneously resulted in a reduction in mean population CVD risk by about $10 \%$ of baseline level. This should translate to a proportional reduction in the incidence of cardiovascular disease events.

The inclusion of nonmodifiable factors in the CVD risk estimator has the potential to bias patient selection towards older males in particular who may have lower modifiable CVD risk than younger patients.

4.4. Limitations. It was not feasible to record all constituent treatments (and compliance with these treatments) offered to the patients included in this research. Thus we were unable to attribute changes observed in blood pressure, total to HDL cholesterol ratio, and smoking habits directly to specific components of the intervention. In addition to this point, the current guideline CVD risk estimator is based predominantly on clinical measures and has limited sensitivity to other aspects of lifestyle modification that might influence CVD risk such as weight loss, increased physical activity, reduced psychosocial stress, avoidance of excessive intake of alcohol, and increased self-esteem that might result from enhanced support from within the community.
The sample population was predominantly male and of older age than would be expected from the profile of CVD events in the local population as a whole. We believe this to be due to an inadvertent sampling bias caused by the inclusion of the nonmodifiable risk factors of gender and age in the CVD risk estimator used. This makes it difficult to generalise beyond the specific population included in this evaluation of the NHS Health Checks programme as implemented in Stoke on Trent.

In assessing the components of absolute CVD risk attributable to each risk factor, we have partitioned the original parametric model into its various components and examined the contribution of each component to the overall risk included in the model. This is an intermediate stage in the actual estimation of absolute CVD risk. Thus, we were unable to attribute changes in risk factors directly to changes in absolute CVD risk estimated.

\section{Conclusions}

The NHS Health Checks programme as implemented in Stoke on Trent was successful in reducing estimated mean population cardiovascular disease risk. Around 59\% of the modifiable risk attributable to the combination of high blood pressure, high cholesterol, and current smoking was removed after one year of intervention.

\section{Conflict of Interests}

The authors declare that there is no conflict of interests regarding the publication of this paper.

\section{Acknowledgments}

The authors are indebted to Linda Picariello and the team of project support workers, Karen Hales, Tracy Pepper, Joanne Fynn, Dianne Machin, and Claire Whitehead, who were responsible for overseeing the recruitment process, contacting patients, and recording outcomes. The role of the clinical champions in supporting and leading implementation within each participating general practice is also acknowledged. Neil Ryder was responsible for coordinating the download of the "at risk" patient lists from electronic medical records in each practice and for coordinating data transfer associated with the project. Marion Beloe and her team of Lifestyle Coaches, Cath Dale (Leisure, Stoke on Trent City Council), and Voluntary Action Stoke on Trent (VAST) were responsible for coordinating enhanced support for lifestyle change within the community. Chris Leese managed the project on behalf of NHS Stoke on Trent and Donna Bailey provided administrative support. This work was supported by Stoke-on-Trent Primary Care Trust as part of the local implementation of the NHS Health Checks programme. The sponsor had input to the design of the study in that the evaluation was to be embedded in primary care and was to take at least the medium term (one year) view. Collection, analysis and interpretation of data, writing of the report, and the decision to submit the paper for publication were entirely the responsibility of the authors. 


\section{References}

[1] K. M. Anderson, P. M. Odell, P. W. F. Wilson, and W. B. Kannel, "Cardiovascular disease risk profiles," American Heart Journal, vol. 121, no. 1, pp. 293-298, 1991.

[2] The Framingham Heart Study, "A Project of the National Heart, Lung and Blood Institute and Boston University," 2013, http:// www.framinghamheartstudy.org/.

[3] C. J. L. Murray and A. D. Lopez, "Alternative projections of mortality and disability by cause 1990-2020: Global Burden of Disease Study," The Lancet, vol. 349, no. 9064, pp. 1498-1504, 1997.

[4] C. D. Mathers and D. Loncar, "Projections of global mortality and burden of disease from 2002 to 2030," PLoS Medicine, vol. 3 , no. 11, article e442, 2006.

[5] S. Yusuf, S. Hawken, S. Ônpuu et al., "Effect of potentially modifiable risk factors associated with myocardial infarction in 52 countries (The INTERHEART Study): case-control study," The Lancet, vol. 364, no. 9438, pp. 937-952, 2004.

[6] Department of Health, Putting Prevention First, Vascular Checks: Risk Assessment and Management, Department of Health, London, UK, 2008.

[7] T. Cochrane, R. Davey, Z. Iqbal et al., "NHS health checks through general practice: randomised trial of population cardiovascular risk reduction," BMC Public Health, vol. 12, no. 1, article 944, 2012.

[8] R. Davey, T. Cochrane, Z. Iqbal et al., "Randomised controlled trial of additional lifestyle support for the reduction of cardiovascular disease risk through primary care in Stoke-on-Trent, UK," Contemporary Clinical Trials, vol. 31, no. 4, pp. 345-354, 2010.

[9] British Cardiac Society, British Hypertension Society, Diabetes UK, Heart UK, Primary Care Cardiovascular Society, and The Stroke Association, "JBS2: Joint British Societies'guidelines on prevention of cardiovascular disease in clinical practice," Heart, vol. 91, supplement 5, pp. v1-v52, 2005.

[10] R. Cooper, J. Cutler, P. Desvigne-Nickens et al., "Trends and disparities in coronary heart disease, stroke, and other cardiovascular diseases in the United States: findings of the National Conference on Cardiovascular Disease Prevention," Circulation, vol. 102, no. 25, pp. 3137-3147, 2000.

[11] P. Barton, L. Andronis, A. Briggs, K. McPherson, and S. Capewell, "Effectiveness and cost effectiveness of cardiovascular disease prevention in whole populations: modelling study," British Medical Journal, vol. 343, no. 7819, Article ID d4044, 2011.

[12] J. Emberson, P. Whincup, R. Morris, M. Walker, and S. Ebrahim, "Evaluating the impact of population and high-risk strategies for the primary prevention of cardiovascular disease," European Heart Journal, vol. 25, no. 6, pp. 484-491, 2004.

[13] E. Vartiainen, "A lot more can be done to prevent cardiovascular diseases," European Heart Journal, vol. 25, no. 6, pp. 457-458, 2004.

[14] S. Ebrahim and J.-P. Casas, "Statins for all by the age of 50 ?" The Lancet, vol. 380, no. 9841, pp. 545-547, 2012.

[15] S. Ebrahim, F. Taylor, K. Ward, A. Beswick, M. Burke, and G. D. Smith, "Multiple risk factor interventions for primary prevention of coronary heart disease," Cochrane Database of Systematic Reviews, no. 1, Article ID CD001561, 2011.

[16] F. Taylor, K. Ward, T. H. M. Moore et al., "Statins for the primary prevention of cardiovascular disease," Cochrane Database of Systematic Reviews, no. 1, Article ID CD004816, 2011.
[17] Association of Public Health Observatories, "Health profile 2011-Stoke-on-Trent-00GL," http://www.apho.org.uk/ resource/item.aspx?RID=105311.

[18] P. Poirier, T. D. Giles, G. A. Bray et al., "Obesity and cardiovascular disease: pathophysiology, evaluation, and effect of weight loss: an update of the 1997 American Heart Association Scientific Statement on obesity and heart disease from the Obesity Committee of the Council on Nutrition, Physical Activity, and Metabolism," Circulation, vol. 113, no. 6, pp. 898918, 2006.

[19] S. Stewart, N. F. Murphy, J. J. V. McMurray, P. Jhund, C. L. Hart, and D. Hole, "Effect of socioeconomic deprivation on the population risk of incident heart failure hospitalisation: an analysis of the Renfrew/Paisley Study," European Journal of Heart Failure, vol. 8, no. 8, pp. 856-863, 2006.

[20] M. Strong, R. Maheswaran, and J. Radford, "Socioeconomic deprivation, coronary heart disease prevalence and quality of care: a practice-level analysis in Rotherham using data from the new UK general practitioner Quality and Outcomes Framework," Journal of Public Health, vol. 28, no. 1, pp. 39-42, 2006.

[21] C. N. B. Merz, J. Dwyer, C. K. Nordstrom, K. G. Walton, J. W. Salerno, and R. H. Schneider, "Psychosocial stress and cardiovascular disease: pathophysiological links," Behavioral Medicine, vol. 27, no. 4, pp. 141-147, 2002.

[22] R. von Känel, "Psychosocial stress and cardiovascular risk: current opinion," Swiss Medical Weekly, vol. 142, Article ID w13502, 2012.

[23] A. V. Diez-Roux, F. J. Nieto, C. Muntaner et al., "Neighborhood environments and coronary heart disease: a multilevel analysis," American Journal of Epidemiology, vol. 146, no. 1, pp. 48-63, 1997.

[24] P. Congdon, "A multilevel model for cardiovascular disease prevalence in the US and its application to micro area prevalence estimates," International Journal of Health Geographics, vol. 8, no. 1, article 6, 2009.

[25] K. Sundquist, M. Malmstro, and S.-E. Johansson, "Neighbourhood deprivation and incidence of coronary heart disease: a multilevel study of 2.6 million women and men in Sweden," Journal of Epidemiology and Community Health, vol. 58, no. 1, pp. 71-77, 2004.

[26] M. T. Cooney, A. Dudina, R. D'Agostino, and I. M. Graham, "Cardiovascular risk-estimation systems in primary prevention: do they differ? Do they make a difference? Can we see the future?" Circulation, vol. 122, no. 3, pp. 300-310, 2010.

[27] G. C. M. Siontis, I. Tzoulaki, K. C. Siontis, and J. P. A. Ioannidis, "Comparisons of established risk prediction models for cardiovascular disease: systematic review," British Medical Journal, vol. 344, no. 7859, Article ID e3318, 2012.

[28] D. Blumenthal, "Performance improvement in health careseizing the moment," The New England Journal of Medicine, vol. 366, no. 21, pp. 1953-1955, 2012. 


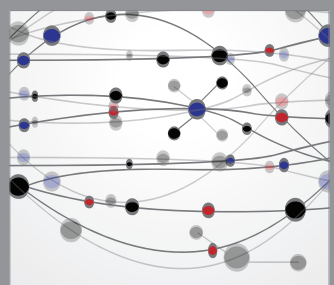

The Scientific World Journal
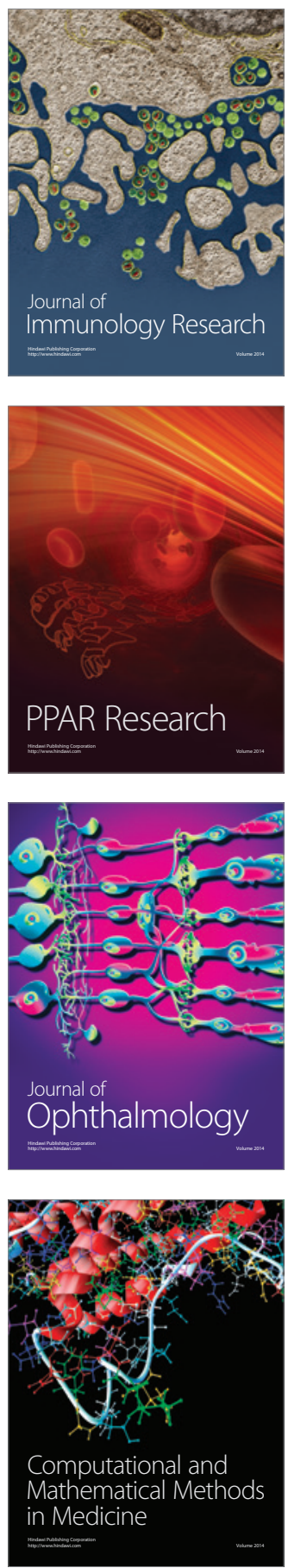

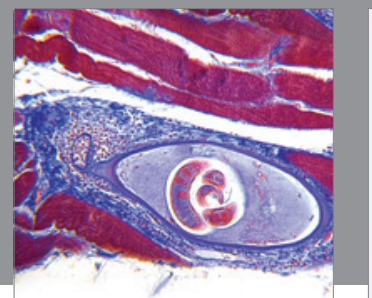

Gastroenterology

Research and Practice
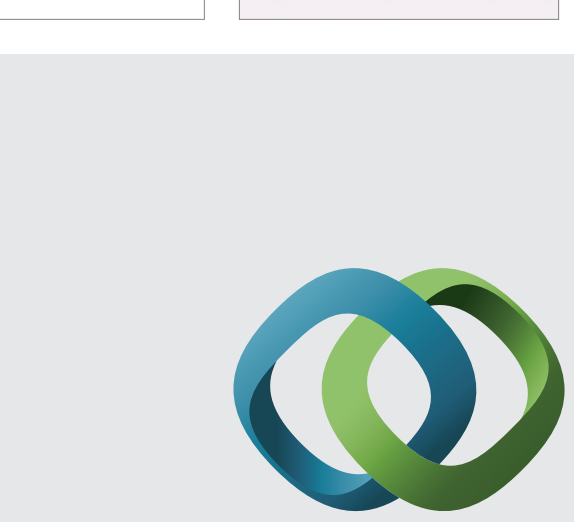

\section{Hindawi}

Submit your manuscripts at

http://www.hindawi.com
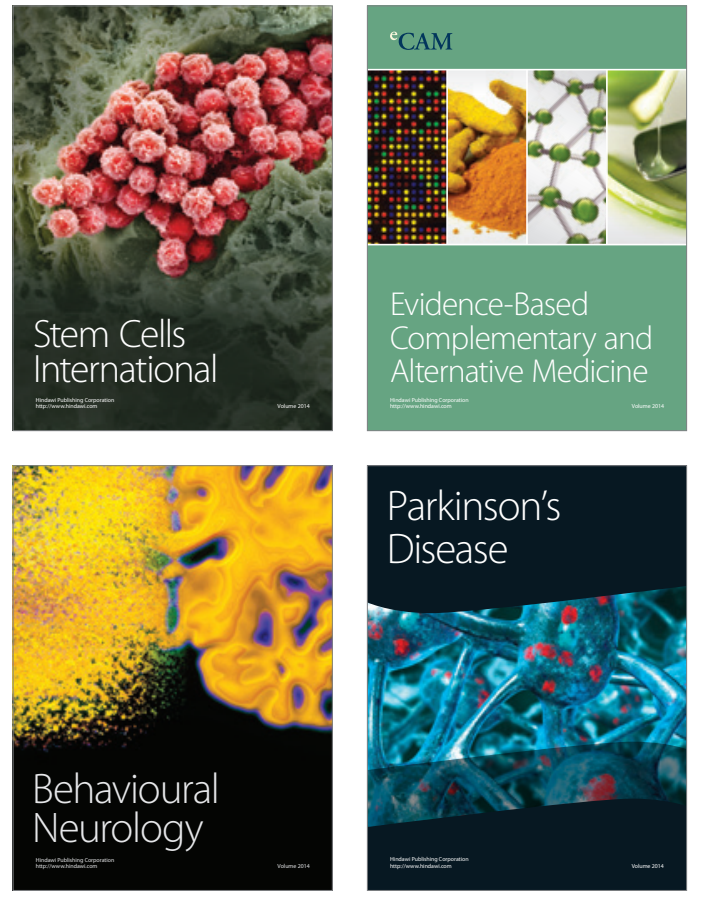
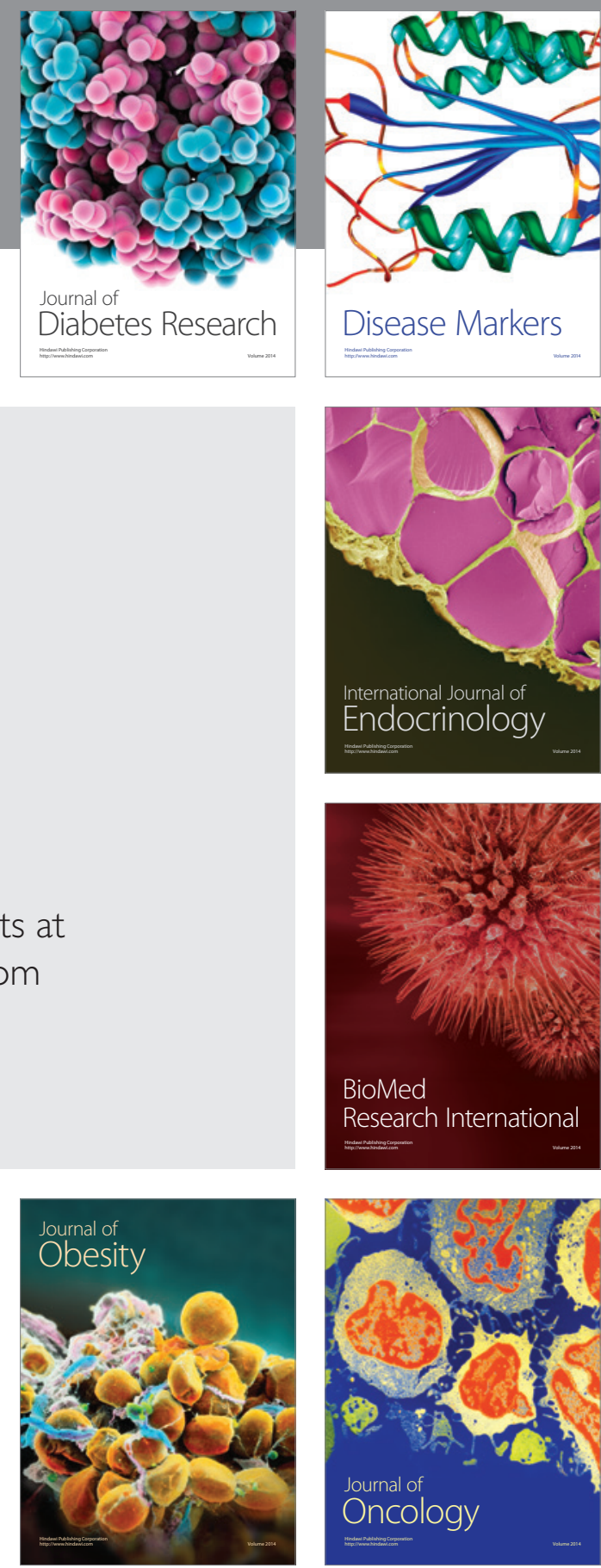

Disease Markers
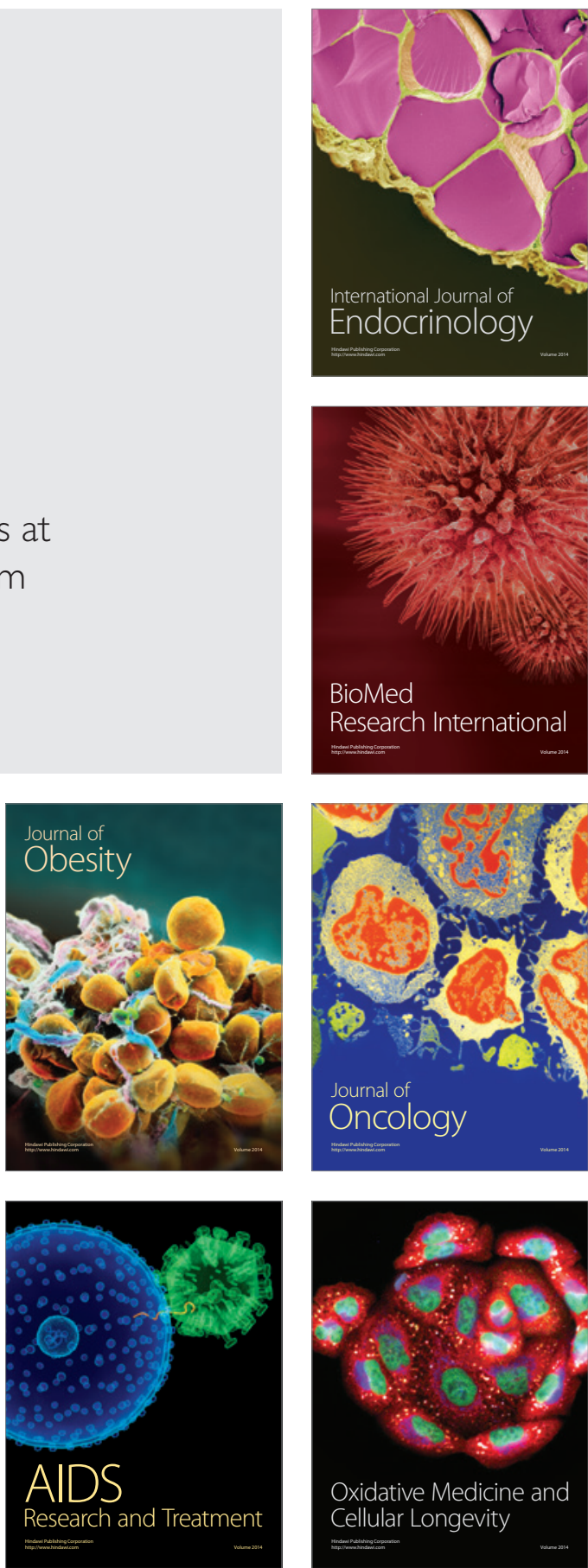